\title{
Topical treatment with water-in-oil ointments improves IL-31 induced impairments of the physical skin barrier and skin barrier function in a 3D atopic dermatitis skin model
}

\author{
Sebastian Huth ${ }^{1}$, Philipp M. Amannn ${ }^{1,2}$, Laurenz Schmitt ${ }^{1}$, Yvonne Marquardt $^{1}$, Ruth Heise ${ }^{1}$, Bernhard Lüscher ${ }^{3}$, Jens M. Baron ${ }^{1}$ \\ 1Department of Dermatology and Allergology, University Hospital RWTH Aachen, Germany \\ ${ }^{2}$ Department of Dermatology, SLK Hospital Heilbronn, Germany \\ ${ }^{3}$ Institute of Biochemistry and Molecular Biology, University Hospital RWTH Aachen, Germany
}

\section{BACKGROUND}

Atopic dermatitis $(A D)$ is a chronic relapsing, pruritic inflammation of the skin with dryness and disturbed skin barrier function and higher risk for allergic sensitization to environmental allergens, affecting $10-20 \%$ of children and $1-3 \%$ adults worldwide with increasing prevalence in highly industrialized countries. A newly described mediator of $A D$ is IL-31, which is strongly expressed in skin samples of AD patients. Recently, we established an IL-31 treated human 3D organotypic AD skin model and showed that IL-31 interferes with the differentiation of primary keratinocytes and inhibits the expression of terminal differentiation markers including filaggrin. In our present study, we used this AD model system to investigate the effects of two different water-in-oil ointments -one of which contains ceramides- on the physical skin barrier structure and function.

\section{MATERIAL AND METHODS}

Cell culture of HaCaT cells with inducible IL-31RA (HaCaT-RA IL31) and normal human dermal fibroblasts (NHDF) and engineering of skin equivalents was done as described previously (Cornelissen et al. J Allergy Clin Immunol 2012;129:426-33). Skin models were cultured over a period of up to 12 days at the air-liquid interphase, and treated every second day with $100 \mathrm{ng} / \mathrm{ml}$ human IL-31. On the sixth day of culture, epidermal part of the models were treated daily with either a basic ointment (BO) or a ceramide-containing ointment (CCO, Sensiderm ${ }^{\circledR}$, Bayer).

The reconstructs were harvested, cut into pieces, and either fixed according to a standardized protocol for routine histology or embedded in Tissue-Tek ${ }^{\circledR}$ O.C.T.TM compound (Sakura Finetek, Zoeterwoude, The Netherlands) for cryosectioning. Parts of the cultures were stored in RNAlater (Ambion/Applied Biosystems, Darmstadt, Germany) for RNA isolation.

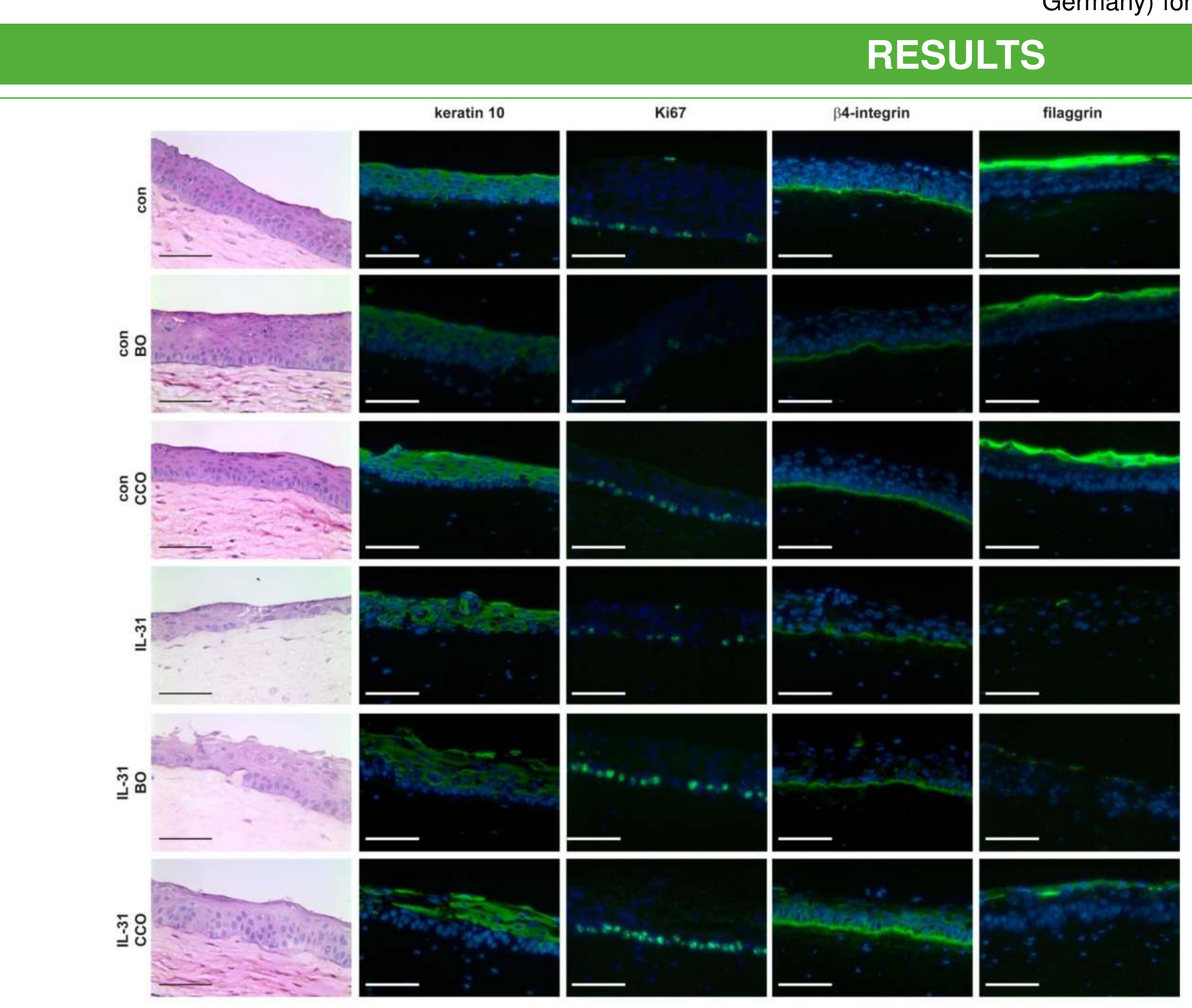

Figure 1. Topical treatment with a ceramide-containing ointment recovered skin barrier and prevented the downregulation of epidermal differentiation markers.

$\mathrm{HE}$ staining and immunofluorescence of a 3D full-thickness AD skin model. Physical skin barrier of the 3D skin model was recovered after daily topical treatment with either BO (basic ointment) or CCO (ceramide-containing ointment) for 6 days. Topical treatment with CCO prevented the downregulation of filaggrin and the disorganisation of other differentiation markers such as keratin 10 or B4integrin. Ki67 protein expression was also found to be upregulated after topical treatment with $\mathrm{CCO}$. 3D models were counterstained with DAPI. con = control; scale bars: $100 \mu \mathrm{m}$

$$
\text { A }
$$
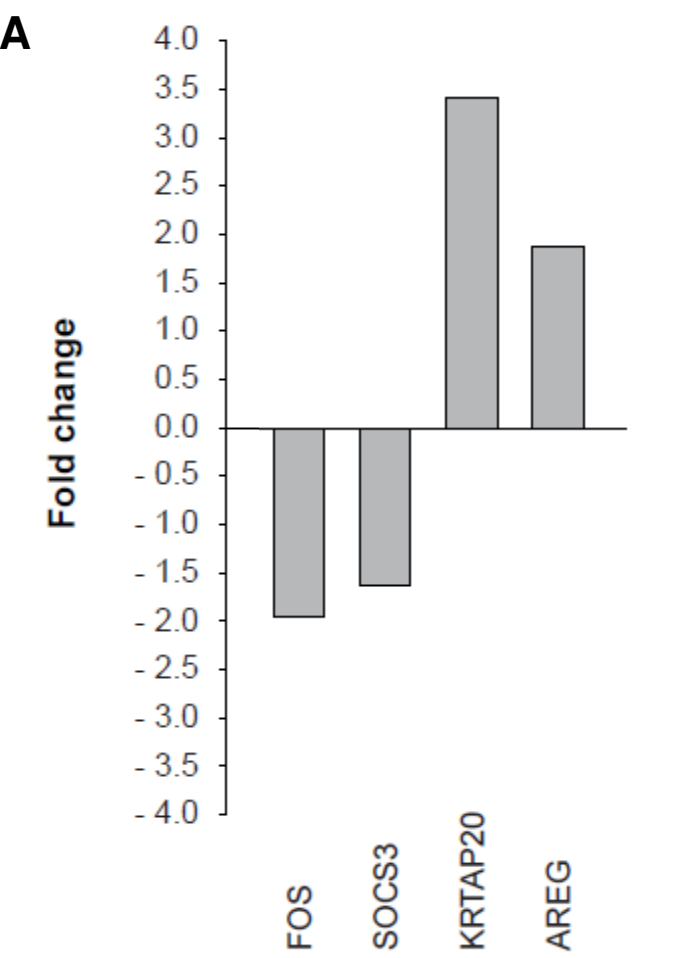

B
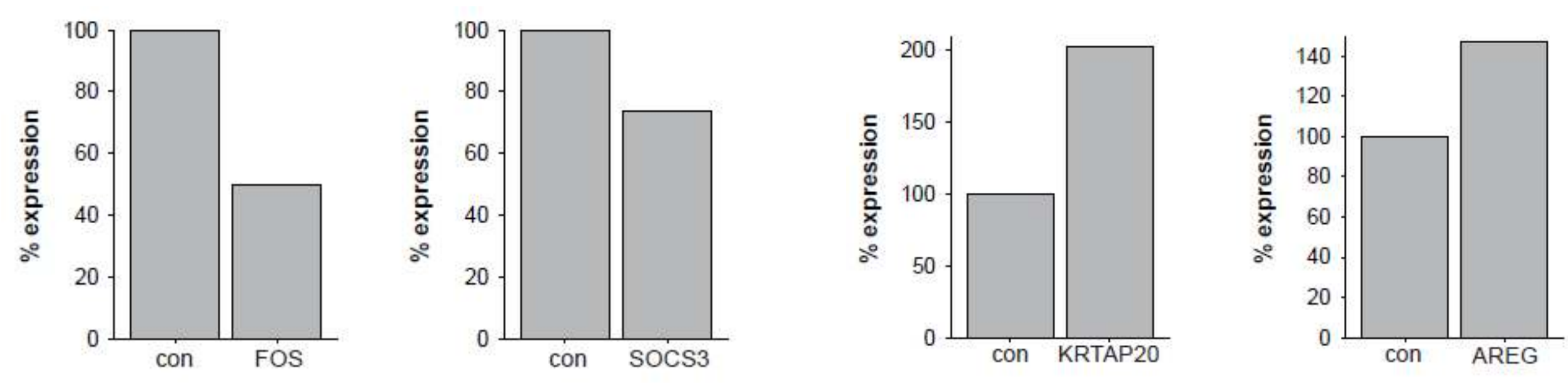

Figure 3. Gene expression in 3D skin models after treatment with a ceramide-containing ointment.

(A) Gene expression was measured using the Affymetrix ${ }^{\circledR}$ Gene Chip Human Exon 2.0 ST array. (B) Gene regulation was confirmed on mRNA level using qRT-PCR analysis. IL-31 treated 3D skin models were harvested after daily topical application of CCO over 6 days. Control IL-31-treated 3D skin models (con) remained topically untreated.

\section{CONCLUSIONS}

First study testing the impact of water-in-oil ointments using an standardized in vitro AD model system.

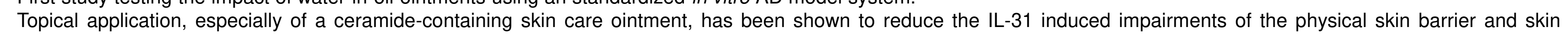
barrier function in this $A D$ in vitro model.

- This 3D AD model can be utilized in future to monitor ex vivo effects of various topical therapies on skin morphology, physiology, and gene expression. 